\title{
Le recours au préformé: une ressource dans l'interaction conversationnelle
}

\author{
Elisabeth Gülich \\ Fakultät für Linguistik und Literaturwissenschaft \\ Universität Bielefeld
}

\section{Introduction}

Un aspect des activités conversationnelles qui jusqu'ici n'a guère retenu l'attention des chercheurs en interaction est celui des routines discursives développées par les interactants pour résoudre des tâches communicatives récurrentes. L'analyse de données orales enregistrées en contexte naturel permet d'observer que les membres d'une société ou d'un groupe social disposent de procédés conventionnels qui mènent à la production de séquences préformées. Ces procédés font partie des «méthodes » conversationnelles pour assurer la production discursive et l'intercompréhension. Les interactants peuvent, s'ils le désirent, exploiter ces ressources; dans certains contextes professionnels ou institutionnels le recours au préformé est (plus ou moins) obligatoire. Notre contribution à cette Table ronde consistera à mettre en lumière quelles sont ces structures, comment les interactants les utilisent et pour l'accomplissement de quelles tâches ils les exploitent. Nous reprenons ici une conception du préformé qui a été développée en collaboration avec Ulrich Krafft et Ulrich Dausendschön-Gay (cf. Dausendschön-Gay/Gülich/Krafft 2007 a et b, Gülich/Krafft 1997).

\section{L'étude du préformé: éléments de théorie et de méthode}

Notre conception du préformé s'inspire largement de l'analyse conversationnelle d'orientation ethnométhodologique ainsi que des recherches que nous avons menées sur le processus de la production discursive et particulièrement sur le travail de la formulation. Le point de départ était l'intérêt pour les expressions idiomatiques dans un corpus de conversations entre interlocuteurs français et allemands, mais l'analyse de ces conversations nous a vite montré que les interactants n'utilisaient guère des expressions idiomatiques au sens étroit du terme, c'est-à-dire des expressions comme «chercher midi à quatorze heures » ou " de pied en cap », alors qu'il y avait d'autres types d'expressions préformées comme par exemple « mettre des vêtements » ou « un œuf à la coque » qui posaient des problèmes de production ou de compréhension aux interlocuteurs (cf. Gülich/Krafft 1992). Ainsi les études de corpus nous ont conduit à élargir progressivement notre conception du préformé et de la préformation : nous qualifions un discours ou segment de discours de préformé si le locuteur s'oriente, pour le produire, sur un modèle (au sens non-normatif de « patron ») disponible dans la communauté discursive. Le locuteur présume que son partenaire connaît et reconnaît le modèle et qu'il l'utilise de son côté à la réception. Cet aspect interactif, largement négligé dans le domaine traditionnel des études sur le préformé, à savoir la phraséologie, est caractéristique d'une approche conversationnelle et nous semble particulièrement important pour comprendre le rôle du préformé dans la conversation.

Le domaine du préformé comprend, outre les expressions phraséologiques au sens restreint du terme (locutions, formules de routine, proverbes), nombre de structures très diverses. Dans la nouvelle encyclopédie de la phraséologie (Burger et al. 2007) les éditeurs ont tenu compte de cet élargissement, tandis que dans la version précédente (Burger et al. 1982) il n'en était pas encore question.

Les structures préformées présentent différents degrés de complexité syntaxique: mots (dans la mesure où ils sont liés à des situations ou contextes précis, par exemple «pardon» ou «bonjour»), syntagmes (comme « au fur et à mesure » ou « chercher midi à quatorze heures »), phrases (les proverbes, les lieux communs, p. ex. «Une hirondelle ne fait pas le printemps», « on fait ce qu'on peut»), unités plus complexes (p. ex. des séquences d'ouverture ou de clôture), textes/discours (comme les discours de salutations et à l'écrit toutes sortes de textes stéréotypés comme les remerciements, les annonces de 
décès, les résumés ou 'abstracts' d'articles dans des revues scientifiques (cf. Gülich 1997, Drescher 1994 et 2002, Gülich/Krafft 1997). La notion de 'genre communicatif' (Bergmann/Luckmann 1995), issue de recherches sociologiques sur des corpus de conversations authentiques, témoigne de la nécessité de tenir compte de processus de préformation au niveau du discours ou des formes conversationnelles complexes. Pour couvrir l'ensemble très diversifié de ces formes, nous utilisons le terme de «structure préformée ». La locution, ou phrasème, est le cas spécifique d'une séquence préformée de la dimension d'un syntagme et stéréotypée au niveau de la mise en mots. Un autre cas spécifique est celui des genres communicatifs qui se caractérisent par des structures générales, servant de cadres, et des cases vides pour les éléments liés à une situation donnée (cf. Gülich/Krafft 1997, cf. aussi les 'schémas' de Grunig 1997 ou le 'modèle locutionnel' de Martin 1997).

Les structures préformées sont spécifiques à des cultures particulières ou liées à des contextes culturels particuliers. Ceci vaut non seulement pour les réalisations linguistiques de ces structures, mail il faut aussi s'attendre à ce qu'elles servent dans différentes cultures à traiter des tâches différentes, et en particulier à ce qu'une culture donnée propose des structures préformées pour traiter certaines tâches, alors que dans une autre culture on ne recourt pas à des solutions conventionnelles pour résoudre les tâches correspondantes. A titre d'exemple on peut citer à une étude comparée sur les compliments en Allemagne et au Caméroun qui montre que là où en Allemagne on a tendance à recourir à des structures préformées on fait des efforts pour trouver des formulations créatives et originales au Caméroun (Mulo Farenkia 2005).

La distinction entre préformé et créatif est cependant une simplification et ne permet pas une catégorisation stricte de toute production discursive. Dans une certaine mesure toute production fait référence à ou s'oriente sur des modèles, mais ces références se distinguent pour être de différents types, plus ou moins manifestes et plus ou moins prévisibles. La question est moins de savoir si telle séquence est préformée ou non que de déterminer en quoi elle l'est et à quel degré. Au lieu d'opposer le recours au préformé à la construction libre on parlera d'une échelle graduée de préformation.

\section{Recours au préformé marqué et recours non-marqué}

Le locuteur peut produire son discours en utilisant des séquences préformées sans les marquer, ou il peut au contraire les marquer, c'est-à-dire les contextualiser comme préformées. Différents procédés conversationnels peuvent servir à marquer une séquence préformée :

Exemple 1 : logés à la même enseigne (Schmale 2007, 46)

M : M. Gaby, marchand grossiste de luminaires ; P: Mlle Papin, marchand de luminaires. Il s'agit de la demande d'un modèle spécifique de lampe et d'une discussion des difficultés économiques du moment.

\begin{tabular}{|c|c|c|}
\hline 2 & M & [oui \de vous laisser tenter $\backslash]$ \\
\hline 3 & $\mathrm{P}$ & eh oui $\backslash$ \\
\hline 4 & & je comprends \\
\hline 5 & $\mathrm{P}$ & en ce moment/ moi j'ai de grosses échéances/ \\
\hline 6 & $\mathrm{M}$ & oui ben on est tous logés à [ la même enseigne vous] savez\ \\
\hline 7 & $\mathrm{P}$ & ) ] \\
\hline 8 & $\mathrm{P}$ & $\mathrm{al}[\mathrm{ors}(\mathrm{on}-)$ \\
\hline 9 & M & [on est TOUS logés à la même enseigne; \\
\hline 10 & $\mathrm{P}$ & je crois $\backslash$ hein (.) bon ben écoutez $\backslash$ je passerai: certainement soit en fin de se(maine), \\
\hline & & soit euh début de l'autre\} $\\
$\hline
\end{tabular}


Dans cet extrait le locuteur $\mathrm{M}$ réagit à une remarque de son interlocutrice $\mathrm{P}(1.5)$ en utilisant l'expression idiomatique « on est tous logés à la même enseigne » (1. 6), qui sert à généraliser l'expérience subjective dont parlait P. («on » et « tous » à la place de «moi je »). Cet emploi est non-marqué. Il est suivi d'un appel à confirmation que le locuteur adresse par «vous savez» à son interlocutrice. Une partie de la réponse de $\mathrm{P}(1.7 / 8)$ est incompréhensible, mais $\mathrm{M}$ n'attend pas sa fin et reformule la séquence préformée en mettant cette fois un accent sur «tous » (1. 9). Ainsi le deuxième emploi est marqué; le procédé de la reformulation fonctionne comme mise en relief. La séquence préformée remplit ainsi une double fonction: Elle souligne le caractère partagé de l'expérience (cf. Drescher 1992 sur le procédé de généralisation) et sert de clôture (cf. Drew/Holt 1988, 1998).

Un procédé fréquent pour marquer l'emploi d'une séquence préformée est le commentaire métadiscursif :

\section{Exemple 2 : les us et coutumes}

Enregistrement d'une consultation à l'Association de Défense des Locataires, 1983 (Bange 1987)

\section{CO : Conseiller; DA : Cliente}

$1 \mathrm{CO}$ alors heu $(($ bégaye $))<$ eu: i vous que heu $>$ si vous avez rien d'écrit/ . i faut considérer que vous

2 avez pas d congél hein donc heu vous dvez partir dans les c- dans les clauses et conditions/..

3 d'un bail verbal/ c'est-à-dire/ c'est c'est trois mois d dédite\ hein . c'est trois mois

4 DA (mais) bon ben jpars/ lmois prochain $\backslash$. non

$5 \mathrm{CO}$ [eh ben oui mais $\backslash$ ou alors heu: 1 - en loi $\mathrm{d}$ quarante huit $\backslash$ quand $\mathrm{y}$ a pas $\mathrm{d}$ bail/ .. j'ai les us

6 DA [mais c-

$7 \mathrm{CO}$ et coutumes qui font: qui font la législation/ heu la: la régle[mentation/ $\mathbf{c}$ 'est $\mathbf{c}$ qu'on appelle

8 DA

[ouais

$9 \mathrm{CO}$ les us et coutumes/ et c'est trois mois $\backslash$. alors i peut vous réclamer trois mois $\mathrm{d}$ loyer $(\ldots)$

Comme dans l'exemple précédent le locuteur reformule la séquence préformée (1. $7 / 8$ : «les us et coutumes ») après l'avoir utilisée de façon non-marquée (1. 5/6). Il introduit la reformulation par un commentaire métadiscursif qui renvoie explicitement au caractère préformé («c'est c qu'on appelle »); celui-ci souligne l'aspect normatif qui est apparemment pertinent pour l'interaction.

L'emploi non-marqué est très fréquent, quand il s'agit par exemple de routines discursives qui servent à structurer la conversation :

\section{Exemple 3: eh tu sais quoi}

(Exemple tiré de Gülich/Mondada, sous presse)

$1 \mathrm{O}$ eh tu sais quoi/ .. on a rencont- tu connais jean noir/

2 qui était dans la classe de ma soeur/ . -fin en d:\

$3 \mathrm{~S} \mathrm{mhm} \mathrm{mhm} \backslash$

$4 \mathrm{O}$ tu sais le grand moitié suisse/ .. [moitié ah non/ . \&

$5 \mathrm{~S} \quad[\mathrm{mhm}$

$6 \mathrm{O}$ \&entièrement suisse/ j'crois ou j'sais plus $\backslash$

$7 \mathrm{~S}$ ouais mais [j'vois qui c'est

$80 \quad$ [en tout cas/ . tu sais il veut faire pilote/

9 et puis euhm: . il a écrit des lettres partout/ et puis

10 . on l'voulait pas/ on l'voulait nulle part/ .. puis 
11 mai'nant i'prend des cours privés de: de pilotage à à

12 l'aéroport là · près d'mulhouse/ . et puis sinon le

13 reste du temps i'travaille/ pa- . seulement par heure/

14 euh dans la euh . à novartis/ . tu sais à bâle/ .

15 et [euh i'fait des trucs avec les ordinateurs/<et\&

$16 \mathrm{~S} \quad[\mathrm{mhm}$

$17 \mathrm{O} \&$ devine qui c'est son patron/ ((en riant) $)>$

$18 \mathrm{~S}<$ müller $(($ bas $))>$

19 ((rires))

$20 \mathrm{O}<$ non $(($ en riant $))>$ non ALberto/

Dans cet extrait il y a différentes séquences préformées qui servent à structurer le développement thématique : Par «eh tu sais quoi» (1. 1) O. introduit un nouveau thème dans la conversation; les hésitations, ruptures et auto-corrections montrent qu'il a une tâche d'organisation complexe à accomplir. Après une séquence secondaire consacrée à l'identification du personnage de jean noir dont il était question au début (1. 4-6) $\mathrm{O}$ reprend le thème par «en tout cas » (8) et produit une brève séquence narrative (8-15), structurée à l'aide de plusieurs emplois de « et puis », « et puis sinon» (1. 9 et 12). Avec «devine qui c'est son patron» il focalise un nouvel aspect et attire l'attention à un point central de l'histoire. On voit ici que les séquences préformées ont des portées différentes concernant la suite de la production discursive : «eh tu sais quoi » a typiquement une portée plus importante, c'est-à-dire le potentiel d'ouvrir une séquence plus large, que par exemple «et puis » avec un potentiel limité à la structuration locale. Mais il faut préciser que la portée effective d'une séquence préformée dans un contexte donné ne dépend pas seulement de son potentiel, mais surtout de la façon dont elle est exploitée par les interactants.

\section{L'orientation interactive sur un modèle}

Si l'on analyse le recours aux structures préformées dans le processus de la production discursive, il faudra prendre en compte différents procédés qui vont de la copie pure et simple du modèle à la complétion de formulaires et à des formes ludiques telles que l'allusion ou la parodie. Contrairement à ce qui se passe dans les citations, que le locuteur marque comme reprises à une source étrangère, il utilise comme siennes les structures préformées. En fait, « citation » et « séquence préformée » ne se distinguent pas parce qu'elles proviennent de sources différentes, mais plutôt en ce que ce sont deux manières d'utiliser des formes préétablies. Cela signifie que les séquences préformées ne sont pas simplement des «préfabriqués» qui seraient reproduits ou réutilisés tel quels dans un discours qui résulte d'une production « libre », mais que s'orienter sur un modèle est aussi un travail de formulation, souvent un travail commun des interlocuteurs. On perçoit ce processus d'orientation et ce travail même quand le locuteur ne connaît le modèle qu'approximativement.

Dans l'extrait suivant l'expression « introduit en erreur » est utilisée à plusieurs reprises comme séquence préformée. Il s'agit d'un entretien téléphonique entre deux interlocutrices, D et E. D appelle E,v qui est membre d'une commission de l'allocation compensatrice, au sujet d'une allocation pour ses parents handicapés qu'elle n'a pas obtenue. D constate au cours de la conversation qu'on ne lui avait pas fourni les informations justes concernant la possibilité de prendre une aide ménagère dans un cas pareil.

Exemple 4 : introduit en erreur (Schmale 2007, 187-190)

$1 \mathrm{E}$ on peut considérer que ça relève d'une allocation aide ménagère $\backslash$ (.) parce que le

2 handicap/ < ((all)) ou la personne a une certaine suffisamment d'autonomie/> pour

3 pouvoir se satisfaire avec une aide ménagère une ou deux heu:res par jou:r\} 
$4 \mathrm{D}$ oui $\backslash$ alors là/ là: ben on nous a introduit en erreur $\backslash$ parce que moi/

$5 \mathrm{E}<(($ all $))$ mais c'est très possiblel>

Ici D utilise l'expression « introduit en erreur » pour la première fois dans l'entretien pour répondre à l'explication fournie par E. E la confirme dans son opinion. Un peu plus tard on retrouve le même type d'échange; E explique dans quel cas on accorde l'allocation compensatrice et D constate qu'on l'a mal informée :

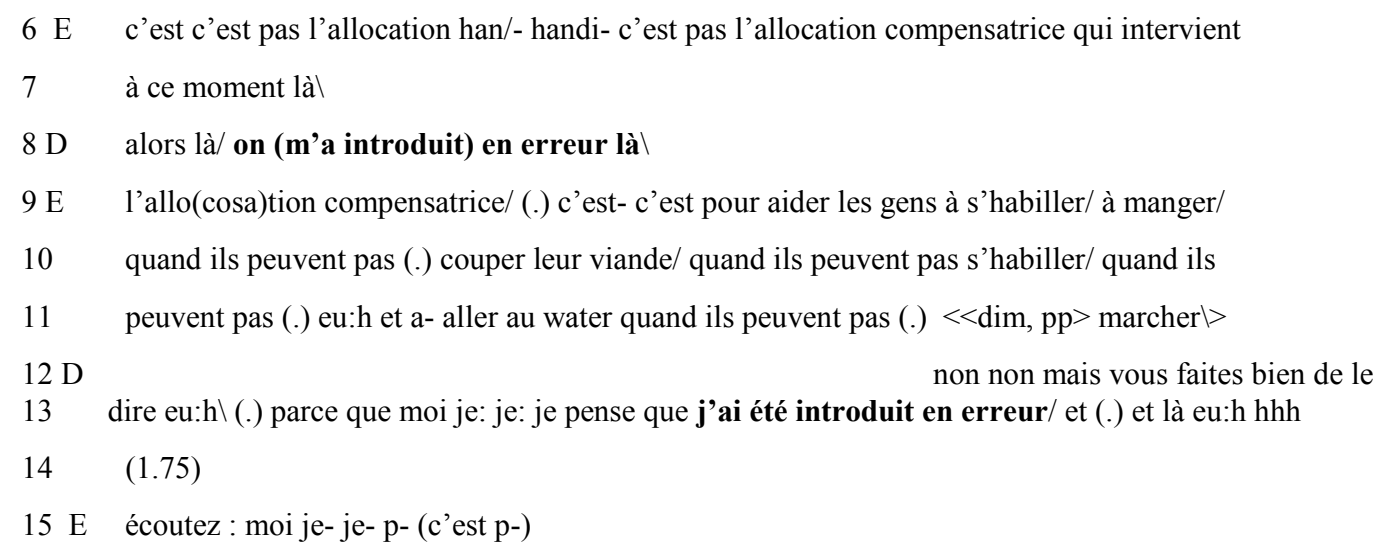

Apparemment pour D « introduit en erreur » est une séquence préformée, appropriée pour formuler un point central dans son argumentation. L'expression 'correcte' telle qu'on la trouve dans le dictionnaire ( « induire en erreur ») sert certainement de 'modèle', mais comme l'interlocutrice E accepte « introduit en erreur » en réagissant au contenu que $\mathrm{D}$ exprime, non à la forme, l'écart entre la forme utilisée et le modèle 'correct' ne joue aucun rôle ni pour l'entretien lui-même, ni pour l'analyse du processus de l'orientation sur le modèle.

Un type de données particulièrement intéressant pour observer le recours à un modèle qui est à la disposition des interlocuteurs et qui leur sert d'orientation, est représenté par l'exemple 5. Il s'agit d'un extrait d'une 'rédaction conversationnelle', c'est-à-dire d'une conversation entre deux interlocuteurs qui rédigent ensemble un texte écrit (cf. p. ex. Krafft / Dausendschön-Gay 1999, de Gaulmyn / Bouchard / Rabatel 2001, Bouchard 2005). En l'occurrence Noël (No), conseiller bancaire, veut rédiger une lettre de confirmation au maire de M. qui lui a proposé un poste ; son amie Barbara (Ba) l'aide. Dans l'extrait qui suit Noël déclenche la recherche d'une formule dont il ne peut que donner une « idée » :

Exemple 5: dans les plus brefs délais (Corpus Dausendschön-Gay/Gülich/Krafft, Bielefeld)

1 No je restE bien entendu à votre entière disposition pour d'autres renseignements/

$2 \quad(16 \mathrm{sec})$

3 No il doit y avoir un tErme qui désigne $: \mathrm{j}$ veux dire eh précisément euh cette idée de :..

$4 \mathrm{~d}$ : de signer le plus tôt possible ce contrat/ pour que : .. pour que tout [soit : soit :

$5 \mathrm{Ba}$ [ouais

6 en règle/

$7 \quad$ No en règle quoil

$8 \quad(23 \mathrm{sec})$

$9 \quad$ No attends/ bouge pas ((va chercher son dictionnaire de synonymes)) (12 sec)

Pour définir l'objet de la recherche les interlocuteurs constatent qu'un modèle existe, qu'il constitue une norme que l'on doit observer pour être «en règle » et qu'il faut donc le trouver. Noël cherche d'abord dans un dictionnaire sous « contrat». Quand Barbara exprime son étonnement, il confirme l'existence du 
modèle : «ouI y a sûrement une expression ». Alors après une longue pause (12 secondes) Barbara propose une séquence préformée qui pourrait résoudre le problème :

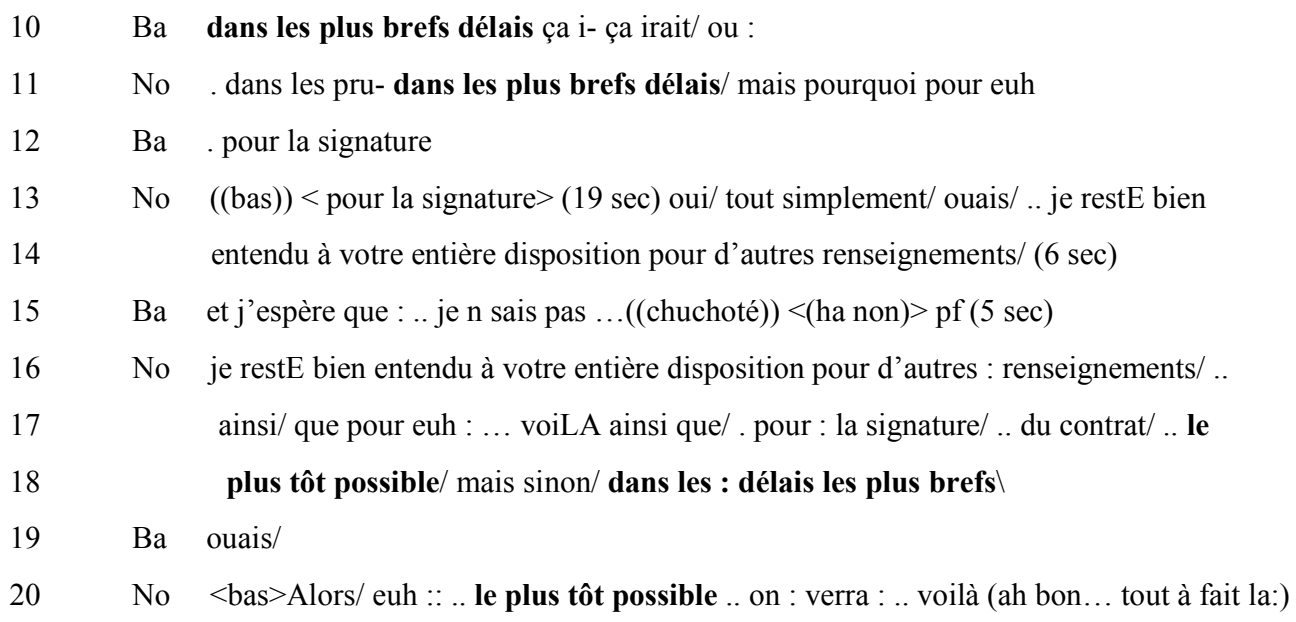

Noël reformule l'expression proposée par Barbara, demande une explication supplémentaire et après une pause assez longue (1. 13) il prend un nouveau départ (« oui/ tout simplement/») et reprend la phrase (1.1) qui avait déclenché la recherche. Barbara entame alors une nouvelle proposition, mais celle-ci reste inachevée (1. 15 : «et j'espère que : »). Noël reformule sa phrase pour la deuxième fois et la complète ensuite en utilisant d'abord la même expression qui lui avait servi au début à donner une «idée » de l'expression recherchée («le plus tôt possible »); ensuite il formule une variante de l'expression proposée par Barbara (1. 18 : dans les : délais les plus brefs »). La formule adéquate est donc négociée entre les interlocuteurs. La décision pour l'une ou l'autre des alternatives est remise à plus tard : « on: verra: » (1. 20). Après avoir trouvé une solution du moins provisoire pour leur problème de formulation les interlocuteurs arrivent aux formules de politesses à la fin de la lettre :

\begin{tabular}{|c|c|c|}
\hline 21 & No & je vous prie d'agréer monsieur nanana l'expression de mes sentiments les \\
\hline 22 & & meilleurs $\backslash$ voilà nfin ça a plein de de phrases de : de po- de formules de politesse/ . \\
\hline 23 & & (parce que) . je vous prie . d'agréer . monsieur le mai :re/ ((ironisant)) bruno : \\
\hline 24 & $\mathrm{Ba}$ & $(($ rit $))$ quoi/ \\
\hline 25 & No & bruno:/ l'expression . de: mes . sentiments . oh les sentimen :ts chais pas (moi) \\
\hline 20 & $\mathrm{Ba}$ & des salutations/ \\
\hline 27 & No & m m m vous prie/ de (monsieur le maire) l'expression/ . non on va laisser comme ça \\
\hline 28 & & ((plus fort)) j préfère laisser comme ça / je : parce que là j'ai l'assurance que c'est un:e \\
\hline 29 & & formule de politesse qui convient très bien/. étant donné que : \\
\hline 30 & $\mathrm{Ba}$ & il l'a mis/ \\
\hline 31 & No & la personne qui l'a rédigée euh travaille dans une administration/ et que là-dessus \\
\hline 32 & & je crois qu'ils sont vraiment pointilleux quoi i : . ils savent quelle formule convient le \\
\hline 33 & & mieux alors \\
\hline 34 & $\mathrm{Ba}$ & $(($ sceptique $))$ ouais \\
\hline 35 & No & non on va pas chercher midi à quatorze heures/ je crois qu c'est bon comme ça/ \\
\hline 36 & $\mathrm{Ba}$ & ouais/ \\
\hline
\end{tabular}

Ici on observe à nouveau un recours explicite aux structures préformées. Déjà le «nanana » à la ligne 21 renvoie au caractère préformé, à une production plus ou moins automatique à cause de laquelle il est 
inutile de continuer, parce que tout le monde connaît la suite de l'expression. On observe également la négociation d'un détail de la formulation («sentiments » vs. «salutations »). En plus, l'orientation sur un modèle qui, dans ce cas-là, est considéré comme normatif, est traité dans un commentaire métadiscursif, qui souligne aussi bien le caractère préformé («ça a plein de de phrases de : de po- de formules de politesse ») que l'aspect normatif : L'argument pour garder «l'expression de mes sentiments... » et non « des salutations » (proposition de Barbara, 1. 26) est justement d'évoquer la compétence d'une personne qui «travaille dans une administration» (1. 31) et qui connaît la norme (1. 32/33 : " ils savent quelle formule convient le mieux alors »). L'orientation sur ce modèle-là est donc consacrée par une autorité. Curieusement le dernier argument pour ne plus chercher d'autres alternatives est formulé à l'aide d'une autre séquence préformée, cette fois une locution idiomatique, qui sert de clôture (cf. Drew/Holt 1988, 1998) : « on va pas chercher midi à quatorze heures ».

\section{Modèles conventionnels et modèles individuels}

Parmi les modèles sur lesquels les interactants s'orientent dans leur production discursive on peut distinguer des modèles conventionnels, partagés par un groupe social, et des modèles individuels, c'est-àdire des structures préformées ou des routines de formulation développées par des locuteurs individuels, quand ils se trouvent fréquemment confrontés aux mêmes tâches communicatives. Les exemples précédents servaient à illustrer le recours aux modèles conventionnels. Les modèles individuels ont à peine attiré l'attention des chercheurs, que ce soit en analyse conversationnelle ou en phraséologie Ils sont effectivement plus difficiles à saisir, car on ne les reconnaît que dans certaines conditions particulières, par exemple quand on dispose - c'est le cas idéal - de données où le même locuteur traite le même sujet ou raconte la même histoire dans des contextes plus ou moins différents. Ainsi Quasthoff (1993) dans une étude sur le développement des compétences narratives chez des enfants a pu observer de tels processus de préformation individuelle, quand les enfants devaient raconter à plusieurs reprises un événement auquel ils avaient assisté. De même dans des interactions entre patients et médecins ou thérapeutes nous avons pu repérer des modèles individuels de description de souffrances ou d'explications de phénomènes médicaux sur lesquels les locuteurs s'orientaient en s'adressant à des interlocuteurs différents (cf. Dausendschön-Gay/Gülich/Krafft 2007 b pour l'analyse de corpus allemands). Mais des processus de stabilisation progressive se trouvent aussi à l'intérieur d'une même conversation. Des exemples de préformation individuelle plus faciles à observer se manifestent dans certains commentaires métadiscursifs comme dans l'extrait suivant d'une conversation radiophonique :

\section{Exemple 6: une grande famille}

(«Allô Macha », France Inter 29.3.1991; Macha Bérenger (MB) avec Jean (J); corpus E. Gülich)

$1 \mathrm{~J}$ parce qu'on participe tellemENt en: fAIt à ces émissions que . de de la nuit/ .. on

2 participe' on écoute les conversations/ puis (un petit peu ....)/ on s trouve intégré/

3 dans une

4 MB c'est vrai qu'on arrive à se connaître un [peu tOUS/ les uns les autres\

[ ouI/ on arrive à connaître y en a

6 certAINs qui RAppellent $\backslash$ alors on les cOnnaît $\backslash$ on se sou[vient/

$8 \mathrm{~J} \quad$ (comme quoi) on dit ben oui/ on connaît celui-là ou celle-là .. y a

$9 \mathrm{MB} \quad$ voilà, c'est eu c'e:st c que j'appelle la

10 famILLe $\backslash$ des [sans-sommeilEs $\backslash$

$11 \mathrm{~J}$ [c'Est la famIlle/ on est int[égré dans une grANde famille`

$12 \mathrm{MB} \quad$ [c'Est la famille/ ((rit)) oui:/ 
$13 \mathrm{~J}$ et par conséquent' donc = euh:, . . bien, chuis content d vous avoir/

Cet échange entre Macha Bérenger et Jean se trouve au début de la conversation, quelques secondes après l'ouverture. Jean, qui s'est tout de suite présenté comme participant habituel à l'émission, décrit son enthousiasme pour le contact téléphonique non seulement avec Macha, mais aussi avec les autres auditeurs qu'on entend parler avec elle. Il semble vouloir résumer ses impressions (1. 1-2) par «on s trouve intégré/ dans une », mais la prise de parole de Macha l'empêche d'achever son énoncé. Macha le confirme («c'est vrai ») en formulant la même idée de la connaissance mutuelle des participants, et Jean la confirme à son tour par une reformulation qu'il enchaîne avant qu'elle n'ait terminé (1. 4 et 5). Tous les deux parlent simultanément; ensuite Jean poursuit sa description. Il y a une brève évaluation simultanée de la part de Macha (1. 7 « c'est bien ça »), qui permet à Jean de continuer. Macha l'interrompt à nouveau par une confirmation («voilà »), après laquelle elle recourt à une séquence préformée qu'elle présente comme un modèle individuel : «c'est eu c'e:st c que j'appelle la famILLe des sans-sommeilEs » (1. 9/10). Elle utilise donc un commentaire métadiscursif, qui ressemble à celui de l'exemple 2, mais à une différence près, le pronom personnel : Dans l'exemple 2 le pronom indéfini «on » dans «c'est: c qu'on appelle » renvoyait à un modèle conventionnel, tandis que le modèle auquel recourt Macha ici est présenté comme un modèle individuel ( «c'est c que j'appelle »). Pour désigner le groupe de ses auditeurs et interlocuteurs habituels elle s'est fabriqué une formule personnelle - du moins la présente-t-elle comme telle.

Ici encore les deux interlocuteurs parlent simultanément, car Jean, sans attendre l'énonciation complète de cette formule, reprend tout de suite le terme de « famille » : «c'Est la famIlle/», et puis il reprend la structure interrompue du début de l'extrait (1. 2/3) et la complète : " on est intégré dans une grANde famille » (1. 11). Macha confirme simultanément : «c'Est la famille» (12). On voit qu'il y a deux modèles concurrents qui servent d'orientation: Jean semble s'orienter sur un modèle conventionnel (des expressions préformées comme 'c'est une grande famille', 'nous sommes tous une grande famille'), Macha par contre s'oriente sur son modèle individuel ('la famille des sans-sommeils'). Mais comme les deux modèles ne se contredisent pas, il y a tout de même une orientation commune, qui se manifeste aussi bien dans la production simultanée que dans l'évaluation positive partagée. Par l'exploitation commune de leurs modèles les deux interlocuteurs se montrent mutuellement combien ils sont d'accord sur la qualité du contact crée par l'émission de Macha.

\section{Conclusion}

Le recours au préformé est une ressource importante dans l'interaction conversationnelle pour différentes raisons, d'abord par sa fréquence: Des recherches quantitatives sur de grands corpus de données orales et écrites en anglais ont montré qu'il y a une moyenne de $55 \%$ des productions discursives qui sont préformées, c'est-à-dire que dans la majorité des cas la décision pour une formulation est déterminée par le «idiom principle », non le «open choice principle» (Erman/Warren 2000 ; pour d'autres résultats quantitatifs cf. Van Lancker-Sidtis/Rallon 2004). On peut supposer que les résultats pour le français ne s'écarteraient pas beaucoup de cette moyenne. Mais l'importance du recours au préformé est avant tout due à son rôle dans la production discursive et dans l'intercompréhension.

Jusqu'à présent les structures préformées, plus exactement: un sous-ensemble des structures préformées, ont surtout été étudié dans la phraséologie, où on s'est intéressé notamment aux aspects lexicaux et grammaticaux. Nos analyses et nos réflexions montrent qu'il est indispensable de tenir compte aussi des phénomènes interactifs et des processus discursifs, si l'on veut comprendre comment fonctionne le recours aux préformé. En ce qui concerne les recherches phraséologiques, notre propos est donc de pratiquer une approche interactionnelle.

Mais notre propos est surtout d'intégrer l'étude du préformé dans les recherches en interaction. Regarder comment les interactants utilisent des séquences préformées, comment ils s'orientent dans leur production sur des modèles qui sont à leur disposition pour résoudre des tâches communicatives permet de mieux comprendre l'interaction conversationnelle et fournit ainsi des éléments supplémentaires pour la décrire plus précisément. Evidemment le recours au préformé est également pertinent pour l'analyse de textes 
écrits, mais l'étude de l'oral a une valeur particulière, parce qu'elle donne accès à l'exploitation interactive du préformé dans le processus de la production et de l'intercompréhension. Car le recours au préformé n'est pas simplement une activité de reproduction d'éléments «préfabriqués » que le locuteur peut préférer ou non à une production libre ou créative (selon un « idiom principle » ou un " open choice principle »), mais il fait partie - tout comme le recours à d'autres procédés de production discursive (p.ex. la reformulation) - du travail de formulation et est soumis à un traitement interactif.

\section{Références}

Bange, P. (éd.) (1987). L'analyse des interactions verbales. La dame de Caluire: une consultation. Bern : Lang.

Bergmann, J. R. / Luckmann, T.(1995). Reconstructive genres of everyday communication. In Quasthoff, U. M. (éd.), Aspects of oral communication. Berlin: de Gruyter, 289-304.

Bouchard, R. (2005). Sources et ressources du discours (académique): Eléments préconstruits et processus de préconstruction en L2. In Bouchard/Mondada, 91-130.

Bouchard, R. / Mondada, L. (éds.) (2005). Les processus de rédaction collective. Paris: L’Harmattan.

Burger, H. / Buhofer, A. / Sialm, A. (éds.) (1982). Handbuch der Phraseologie. Berlin, New York: de Gruyter.

Burger, H. / Dobrovol'skij, D. / Kühn, P. / Norrick, N.R. (éds.) (2007). Phraseologie. Ein internationales Handbuch zeitgenössischer Forschung. Berlin, New York: de Gruyter.

Dausendschön-Gay, U. / Gülich, E. / Krafft, U. (2007a). Phraseologische/formelhafte Texte. In Burger et al., vol. I, 468-481.

Dausendschön-Gay, U. / Gülich, E. / Krafft, U. (2007b). Vorgeformtheit als Ressource im konversationellen Formulierungs- und Verständigungsprozess. In Hausendorf, H. (éd.): Gespräch als Prozess. Linguistische Aspekte der Zeitlichkeit verbaler Interaktion. Tübingen: Narr, 181-219.

Dausendschön-Gay, U. / Krafft, U. (1991). Tâche conversationnelle et organisation du discours. In DausendschönGay, U. / Gülich, E. / Krafft, U. (éds.), Linguistische Interaktionsanalysen. Beiträge zum 20. Romanistentag Freiburg 1987. Tübingen: Niemeyer, 131-154.

Drescher, M. (1992). Verallgemeinerungen als Verfahren der Textkonstitution. Untersuchungen zu französischen Texten aus mündlicher und schriftlicher Kommunikation. Stuttgart: Franz Steiner.

Drescher, M. (1994). „Für zukünftige Bewerbungen wünschen wir Ihnen mehr Erfolg“. Zur Formelhaftigkeit von Absagebriefen. In Deutsche Sprache 22, 117-137.

Drescher, M. (2002). Theoretische und methodische Aspekte eines kontrastiven Textsortenvergleichs am Beispiel französischer und spanischer Todesanzeigen. In Drescher, M. (éd.), Textsorten im romanischen Sprachvergleich. Tübingen: Stauffenburg, 41-61.

Drew, P. / Holt, E. (1988). Complainable Matters: The use of idiomatic expressions in making complaints. In Social Problems 35, 398-417.

Drew, P./ Holt, E. (1998). Figures of Speech: Figurative expressions and the management of topic transition in conversation. In Language in Society 27, 495-522.

Erman, B. / Warren (2000). The idiom principle and the open choice principle. In Text 20 (1), 29-62.

de Gaulmyn, M.M. / Bouchard, R. / Rabatel, A. (éds.) (2001). Le processus rédactionnel. Ecrire à plusieurs voix. Paris : L'Harmattan.

Grunig, B.-N. (1997). La locution comme défi aux théories linguistiques : une solution d'ordre mémoriel? In Martins-Baltar (éd.), 225-240.

Gülich, E. (1997). Routineformeln und Formulierungsroutinen. Ein Beitrag zur Beschreibung formelhafter Texte. In Wimmer, R. /Berens, F.-J. (éds.), Wortbildung und Phraseologie. Tübingen: Narr, 131-175.

Gülich, E./Krafft, U. (1992). 'Ich mag es besser' - Konversationelle Bearbeitung vorgeformter Ausdrücke in Gesprächen zwischen deutschen und französischen Sprechern. In Fremdsprachen Lehren und Lernen 21, 65-87. 
Gülich, E. /Krafft, U. (1997). Le rôle du «préfabriqué » dans les processus de production discursive. In MartinsBaltar, 241-276.

Gülich, E./ Mondada, L. (2001). Analyse conversationnelle. In Holtus, G. / Metzeltin, M. / Schmitt, C. (éds.) (2001), Lexikon der Romanistischen Linguistik (LRL), vol. I,2: Methodologie. Tübingen: Niemeyer, 196-250.

Gülich, E. / Mondada, L. (sous presse): Konversationsanalyse. Eine Einführung am Beispiel des Französischen. Tübingen: Niemeyer (Romanistische Arbeitshefte).

Krafft, U. / Dausendschön-Gay, U. (1999). Système écrivant et processus de mise en mots dans les rédactions conversationnelles. In Langages, juin, 51-67.

Martin, R. (1997). Sur les facteurs du figement lexical. In Martins-Baltar, 291-305.

Martins-Baltar, M. (éd.) (1997). La locution entre langue et usages. Fontenay/Saint Cloud : ENS Editions.

Mejri, S. (2007). French phraseology. In Burger et al., vol. II, 682-691.

Mulo Farenkia, B. (2005). Kreativität und Formelhaftigkeit in der Realisierung von Komplimenten: Ein deutschkamerunischer Vergleich. In Linguistik online 22, 1.

Quasthoff, U. M. (1993). Vielfalt oder Konstanz in den sprachlichen Formen des Kindes. Linguistische, entwicklungstheoretische und didaktische Aspekte. In Der Deutschunterricht VI, 44-56.

Schmale, G. (éd.) (2007). Communications téléphoniques II: Conversations en contexte professionnel et institutionnel. Un corpus de transcriptions. Beiträge zur Fremdsprachenvermittlung, Sonderheft 12.

Stein, S. (1995). Formelhafte Sprache. Untersuchungen zu ihren pragmatischen und kognitiven Funktionen im gegenwärtigen Deutsch. Frankfurt/M.: Lang.

Stein, S. (2004). Formelhaftigkeit und Routinen in mündlicher Kommunikation. In Steyer, 262-288.

Steyer, K. (éd.) (2004). Wortverbindungen - mehr oder weniger fest. Berlin: de Gruyter.

Tannen, D. (1987). Repetition in conversation: Toward a poetics of talk. In Language 63, 574-605.

Van Lancker-Sidtis, D. / Rallon, G. (2004) . Tracking the incidence of formulaic expressions in everyday speech : methods for classification and verification. In Language \& Communication 24, 3, 207-240.

\section{Conventions de transcription}

(Les conventions de transcription ont été reprises de Gülich/Mondada, sous presse)

(.) micropause

(2.3) pause chronométrée en secondes

... ... pauses (le nombre des point indique la durée estimée)

(2 s) pauses en secondes

[ ] chevauchements (début et fin)

$=\quad$ enchaînement rapide

/ I intonation montante/ descendantel

: allongement vocalique

$\wedge$ liaison

exTRA segment accentué

${ }^{\circ}$ bon $^{\circ} \quad$ volume bas, murmuré de la voix

par- troncation

.h aspiration du locuteur

h expiration du locuteur

((rire)) commentaires du transcripteur et description de phénomènes non transcrits 
ISBN 978-2-7598-0358-3, Paris, 2008, Institut de Linguistique Française

Discours, pragmatique et interaction

DOI $10.1051 / \mathrm{cmlf0} 8315$

$<>\quad$ délimitation des phénomènes entre (( ))

\& continuation du tour de parole

(il va) essai de transcription d'un segment difficile à identifier

xxx segment inaudible

* * délimitation des phénomènes multimodaux 